\title{
Prevention of venous thromboembolism in Wales: results of a survey among general surgeons
}

\author{
E V Williams, R S Williams, J L Hughes, K L Williams, M E Foster, M H Lewis
}

Postgrad Med J 2002;78:88-91

See end of article for authors' affiliations

.....................

Correspondence to: Mr E V Williams,

Department of Surgery,

Royal Glamorgan Hospital

Ynysmaerdy, Llantrisant

CF72 8XR, UK; eifion@

vwilliams.freeserve.co.uk

Submitted

28 November 2000

Accepted 3 July 200

\begin{abstract}
Objective: To examine the current attitudes towards the prevention of venous thromboembolism among a cohort of surgeons.

Design: A postal survey, comprising a questionnaire covering various aspects of venous thromboembolism prophylaxis was sent to all $(n=84)$ consultant general surgeons in Wales.

Results: Replies were received from 57 surgeons $(68 \%)$, all of whom routinely used prophylaxis, the most frequent modalities used being heparin (100\%) and graded compression stockings $(79 \%)$. A combination of physical and pharmacological methods was used by over $89 \%$ of surgeons, with $60 \%$ starting prophylaxis more than two hours before operation. All surgeons continued prophylaxis after surgery, $53 \%$ until patients were mobile, $45 \%$ until they were discharged, and one surgeon continued prophylaxis for seven days after discharge. The thrombosis risk factors considered most important by surgeons when deciding about prophylaxis were (i) a previous history of venous thromboembolism, (ii) hypercoagulability, and (iii) malignancy.

Conclusions: This study confirms that Welsh surgeons conform to standard methods, but also highlights some uncertainties that are present in current surgical practice. Those who responded all routinely used prophylaxis, the timing of which was variable. The main risk factors identified when considering prophylaxis were previous history of deep vein thrombosis/pulmonary embolism, hypercoagulability, and the presence of malignancy. Suggestions for future practice are made.
\end{abstract}

Dostis ostoperative venous thromboembolism represents a serious threat to patients undergoing a surgical procedure. Without antithrombotic prophylaxis, up to $15 \%$ of patients after major abdominal surgery develop a deep vein thrombosis (DVT), with the risk of fatal pulmonary embolism approaching $1 \%{ }^{1}$

The prevalence of venous thromboembolism will probably increase in the future as the age of the population is increasing, more elderly patients are admitted for major surgical procedures, and many patients, young and old, are discharged from surgical wards (including day surgery) before they are fully ambulant.

In the past 20 years, many studies have shown that several prophylaxis modalities (pharmacological and physical) have significantly reduced the rate of postoperative venous thromboembolism. ${ }^{1-5}$ Despite this, there is a worldwide variation in practice among surgeons. The purpose of this study was to assess the current attitudes towards postoperative prevention of venous thromboembolism in a national survey of busy general surgeons.

\section{SUBJECTS AND METHODS}

A two page questionnaire (fig 1) was designed by EVW in collaboration with a statistician to ensure its validity. It was then sent to all practising general surgeons in Wales between January and April 1999. The survey consisted of 10 questions regarding awareness and risk factors for venous thromboembolism, the timing and duration of prophylaxis, together with preferred modalities for general and specific clinical situations. The questionnaires were dispatched with a covering letter explaining the purpose of the survey.

All replies were received anonymously and were tabulated into an Excel database. Statistical analysis was carried out using SPSS 6.0 for Windows (SPSS, Chicago, Illinois, USA). The results for risk factor assessment (question 6) were determined from the scores (where 1 was the most important and
9 the least important values) given by each surgeon and the total score (sum) was calculated. The risk factors with the lowest means were then considered the most important indicators for prophylaxis. Non-parametric analysis of data for risk factor assessment was performed using the Wilcoxon signed rank test. Kendall's W test was used to determine the degree of agreement between the surgeon's rankings.

\section{RESULTS}

Replies were received from 57 surgeons, a response rate of $68 \%$. All routinely used prophylaxis, 100\% using heparin (unfractionated and/or low molecular weight (LMWH)) and over $89 \%$ utilising a combination of both pharmacological and physical methods (table 1 ). Six surgeons (11\%) used only one modality (heparin), 19 (33\%) used two modalities, and the remaining $32(56 \%)$ used a combination of three or more methods.

With regard to timing, all surgeons started prophylaxis before surgery, one surgeon (2\%) started prophylaxis on the patient's admission to hospital, $34(60 \%)$ more than two hours before operation, and the remaining 22 (39\%) started prophylaxis with the premedication. All surgeons continued prophylaxis after surgery, 30 (53\%) until the patient was mobile, and $25(43 \%)$ until the patient was discharged. One surgeon continued prophylaxis for seven days after discharge, and another would continue prophylaxis in high risk cases for 14 days after discharge.

The most common diagnostic investigation made to confirm a suspected DVT was B-mode ultrasonography (58\%). Forty per cent of surgeons used a combination of contrast venography and ultrasonography, and $2 \%$ used duplex scanning.

Abbreviations: DVT, deep vein thrombosis; LMWH, low molecular weight heparin 

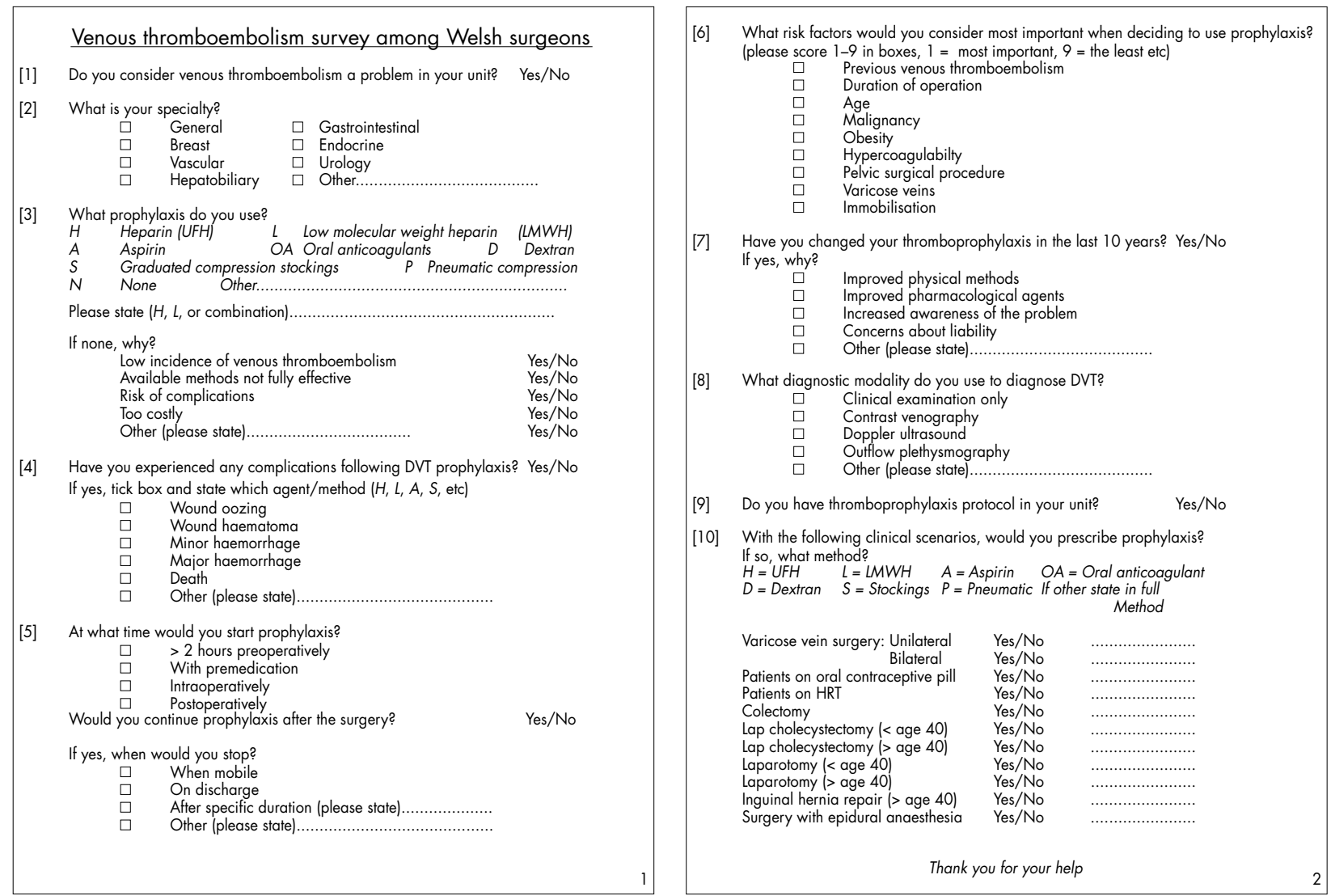

Figure 1 Questionnaire sent to general surgeons in Wales (HRT, oestrogen replacement therapy).

\begin{tabular}{|ll|}
\hline $\begin{array}{l}\text { Table } 1 \\
\text { surgeons }\end{array}$ & Modalities of prophylaxis used by Welsh \\
\hline Modality & No (\%) \\
\hline Elastic stockings & $45(79)$ \\
Pneumatic compression & $31(54)$ \\
Unfractioned heparin (UFH) & $33(58)$ \\
Low molecular weight heparin (LMWH) & $17(30)$ \\
Combined UFH and LMWH & $7(12)$ \\
Aspirin & $2(4)$ \\
Dextran & $1(2)$ \\
Warfarin & $1(2)$ \\
\hline
\end{tabular}

Twenty three $(40 \%)$ surgeons reported problems after prophylaxis, with wound oozing and haematoma appearing as the most frequent. The death of three patients has also been attributed (through the anonymous questionnaires) directly to venous thromboembolism prophylaxis.

The main risk factors contributing to surgeons describing a "high risk" state were a previous history of venous thromboembolism, hypercoagulibilty, and malignancy (table 2). The degree of agreement between the surgeons is very good and these variables were found to be highly significant.

Table 3 shows the results regarding the various clinical scenarios.

All respondents were general surgeons with a variety of subspecialty interests working mainly in district general hospitals. Over half $(51 \%)$ considered that venous thromboembolism was a potential problem in their unit, but regardless of this nine surgeons (16\%) stated that they did not have a departmental protocol. During the past 10 years only 26 surgeons $(46 \%)$ have modified their approach to prophylaxis for venous thromboembolism, the most common reasons for doing so was the availability of improved pharmacological methods and increased awareness of the problems.

\section{DISCUSSION}

This survey has shown that current prophylactic measures are universally employed by all responding Welsh surgeons. The preferred thromboprophylactic modalities utilised were heparin, graded compression stockings, and intermittent pneumatic compression (table 1). These results are similar to those found in Australia and New Zealand ${ }^{6}$ and in America ${ }^{7}$ with the high implementation of physical methods. All Welsh surgeons used heparin (in one form or other), with less than half using LMWH. The reasons for this poor uptake are not clear, it may be related to cost, as LMWH is certainly more expensive than the conventional unfractionated heparins. There seems little doubt that the LMWHs are the most convenient of the pharmacological methods to administer: they are given once daily and require no laboratory monitoring. We feel that there should be a wider adoption of LMWH for prophylaxis, which is justified on the basis of greater safety, patient acceptability, and saving of nursing time. ${ }^{8}$ It is also surprising to note that two surgeons are still using aspirin. This modality has been found to be ineffective in preventing venous thromboembolism in general surgical patients, and is therefore not recommended as an appropriate strategy. ${ }^{9}$

All surgeons as expected start prophylaxis before surgery, with the majority starting it more than two hours before operation. There appears to be no adverse consequence of giving the first dose two hours before operation, ${ }^{10}$ and there may be an additional benefit of preventing DVT developing during operation or in the immediate postoperative period. However high risk patients (where higher doses are required) would probably benefit more if prophylaxis started earlier (10-12 
Table 2 Risk factors that are considered most important indicators for prophylaxis for venous thromboembolism (VTE)

\begin{tabular}{llllll}
\hline Risk factor & No & $\begin{array}{l}\text { Total score } \\
\text { given }\end{array}$ & Mean & SD & $\begin{array}{l}\text { Kendall's W test } \\
\text { mean rank }\end{array}$ \\
\hline Previous VTE & 40 & 52 & 1.30 & 0.82 & 1.36 \\
Hypercoagulability & 36 & 113 & 3.13 & 1.69 & 3.30 \\
Malignancy & 38 & 140 & 3.68 & 1.93 & 3.96 \\
Pelvic surgical procedure & 38 & 175 & 4.61 & 1.97 & 4.79 \\
Duration of operation & 36 & 190 & 5.28 & 2.12 & 5.36 \\
Obesity & 38 & 206 & 5.42 & 1.95 & 5.70 \\
Immobility & 36 & 203 & 5.64 & 2.19 & 5.76 \\
Age & 37 & 222 & 6.00 & 2.05 & 6.30 \\
Varicose veins & 36 & 293 & 8.14 & 1.81 & 8.39 \\
\hline No, number of respondents. & \\
Kendall's coefficient of concordance, W=0.531, p=0.000. &
\end{tabular}

\begin{tabular}{|c|c|c|c|c|c|c|}
\hline Clinical scenario & $\begin{array}{l}\text { No } \\
\text { prophylaxis }\end{array}$ & No answer & $\begin{array}{l}\text { Prophylaxis } \\
\text { given }\end{array}$ & Pharmacological & Physical & Combination \\
\hline Unilateral varicose veins & 63 & 7 & 30 & 13 & 7 & 10 \\
\hline Bilateral varicose veins & 49 & 7 & 44 & 22 & 4 & 18 \\
\hline Patients on $\mathrm{OCP}$ & 5 & 5 & 90 & 44 & 2 & 44 \\
\hline Patients on HRT & 23 & 4 & 73 & 33 & 0 & 40 \\
\hline Colectomy & 4 & 4 & 92 & 35 & 2 & 55 \\
\hline Lap chole $<40$ & 35 & 4 & 61 & 19 & 10 & 32 \\
\hline Lap chole $>40$ & 9 & 3 & 88 & 32 & 6 & 50 \\
\hline Laparotomy $<40$ & 18 & 5 & 77 & 32 & 3 & 42 \\
\hline Laparotomy $>40$ & 4 & 5 & 91 & 35 & 2 & 54 \\
\hline Hernia repair & 49 & 4 & 47 & 19 & 10 & 18 \\
\hline Epidural anesthesia & 37 & 9 & 54 * & 14 & 13 & 17 \\
\hline
\end{tabular}

hours before an operation), in order to avoid excessive intraoperative bleeding. ${ }^{10}$ The restoration of mobility is seen as the commonest indication for stopping prophylaxis. There is evidence that the thrombotic risk persists for many weeks after surgery, ${ }^{11}$ despite this prophylaxis being stopped by the majority within days of operation.

Regarding risk factor assessment, it is no surprise that previous venous thromboembolism is the most important indicator for prophylaxis, as several studies have shown that the incidence of postoperative DVT in these patients is over $50 \% .{ }^{12}{ }^{13}$ What is surprising, however, is the low percentage who consider age as an important risk factor, particularly as several studies have shown an association between advancing age and increased risk of venous thromboembolism. ${ }^{13}{ }^{14}$

It is interesting to assess the surgeons' opinions in specific clinical situations, where a noticeable trend is the combined use of pharmacological and physical modalities, with only a few having a preference for physical methods alone. A major concern, however, is that two surgeons (4\%) would perform a colectomy without prophylaxis. There has been an association between varicose veins and DVT, where a number of studies have shown an increased risk of calf DVT. ${ }^{13}{ }^{14}$ It is therefore no surprise that $30 \%$ of Welsh surgeons routinely recommend prophylaxis for patients undergoing unilateral varicose vein surgery. This figure is far greater than the $12 \%$ stated in a recent survey of vascular surgeons. ${ }^{15}$

Whether to stop the oral contraceptive pill before major surgery is still a controversial issue. ${ }^{16}{ }^{17}$ Ninety per cent of surgeons would give prophylaxis to those on the pill, which is probably a reflection of their concerns over these uncertainties. In contrast to the oral contraceptive pill, there are no reliable data to suggest that postmenopausal oestrogen replacement therapy increases the risk of venous thromboembolism. ${ }^{18} 19$ It is therefore surprising that $73 \%$ of surgeons would recommend prophylaxis for patients on oestrogen replacement therapy. As expected, fewer surgeons would adopt prophylaxis for hernia repair, as the duration of current surgical practice is short and venous thromboembolism risk minimal. With the use of epidural anaesthesia, 37\% of surgeons would not adopt prophylaxis, with the obvious concerns about haemorrhage, leading to spinal cord compression. However it has been shown that provided adequate precaution is taken, epidural anaesthesia can be safely used in patients receiving anticoagulant therapy. ${ }^{20}$ We suspect that most surgeons would regard this as an anaesthetic issue and would therefore do whatever their anaesthetist instructs.

With regard to the numerous $(40 \%)$ complications sustained, these are merely speculative remarks on behalf of the surgeons. Unfortunately in the absence of evidence, no conclusions can be drawn.

This survey shows good evidence on the practice of consultant surgeons in Wales, and allows comparisons with other surgeons throughout the world. It also confirms the uncertainties that are present in today's current surgical practice. This type of survey has several limitations. Our response rate of $68 \%$ is favourable, however it is possible (but unlikely) that the $32 \%$ of the non-responders were antiprophylaxis, and hence would have given a totally different complexion to our results. It is also important to realise that although all surgeons state that their use of prophylaxis is universal, this does not always equate to the proportion of patients who actually receive prophylaxis. Despite these limitations, some suggestions for future practice are listed (box 1).

Finally consideration for using a "default" system should be encouraged. One possible method is that absolutely everybody gets prophylaxis, which could become an integral part of the treatment chart. All patients would therefore receive heparin unless it is actively crossed off. So, the "fail safe" situation is that all patients are given heparin and not that they are not given heparin. If such a policy is introduced, then vigilance is 


\section{Box 1: Suggestions for future practice}

- All surgical units to establish departmental protocols.

- A risk assessment chart to be evaluated for each patient on admission.

- All patients should receive prophylaxis according to their risk, based on the THRIFT guidelines. ${ }^{4}$

- Consider pharmacological (LMWH) and physical methods of prophylaxis.

- Duplex ultrasound should be the principal tool to investigate for DVT.

- Encourage early postoperative mobilisation.

- Reasons not to give/withdraw prophylaxis should be fully communicated and recorded.

- Audit of surgical practice is essential.

needed to exclude those patients with a bleeding tendency, active peptic ulceration, severe hypertension, heparin induced thrombocytopenia, or known hypersensitivity to heparin.

\section{CONCLUSION}

The results of this survey indicate that Welsh surgeons conform to standard practice, as all the respondents routinely use prophylactic measures with over $89 \%$ using a combination of pharmacological and physical methods. Venous thromboembolism is considered a major problem and over $84 \%$ of surgeons adhere to protocols that are available in their units. Worryingly and potentially with medicolegal implications is that nine surgeons (16\%) do not have a departmental protocol. The main risk factors when considering prophylaxis are previous venous thromboembolism, hypercoagulability, and malignancy.

\section{ACKNOWLEDGMENTS}

We would like to thank all the participating consultant surgeons who took their time to complete and return the questionnaire. We are also grateful to Miss Nicola Richardson for her assistance in the dispatch and collection of the questionnaires, and also to Dr T B Vaughan for his initial statistical advice.

\section{Authors' affiliations}

E V Williams, R S Williams, J L Hughes, M E Foster, M H Lewis, Department of Surgery, Royal Glamorgan Hospital, Ynysmaerdy, Llantrisant, UK

K L Williams, Department of Medical Statistics, University of Wales College of Medicine, Cardiff, UK

\section{REFERENCES}

1 Clagett GP, Reisch JS. Prevention of venous thromboembolism in general surgical patients. Ann Surg 1988;208:227-40.

2 Collins $\mathbf{R}$, Scrimgeur R, Yusuf A, et al. Reduction in fatal pulmonary embolism and venous thrombosis by perioperative administration of subcutaneous heparin. N Engl J Med 1988;318:1162-73.

3 Colditz GA, Tuden RL, Oster G. Rates of venous thrombosis after general surgery: combined results of randomised clinical trials. Lancet 1986;ii: 143-6.

4 Thromboembolic Risk Factors (THRIFT) Consensus Group. Risk of and prophylaxis for venous thromboembolism in hospital patients. BM 1992;305:567-74.

5 Clagett GP, Anderson FA Jr, Geerts W, et al. Prevention of venous thromboembolism. Chest 1998;114:531S-60S.

6 Fletcher JP, Koutts J, Ockelford PA. Deep vein thrombosis prophylaxis: a survey of current practice in Australia and New Zealand. Aust N Z J Surg 1992;62:601-5.

7 Caprini JA, Arceulus Jl, Hoffman K, et al. Prevention of venous thromboembolism in North America: results of a survey among general surgeons. J Vasc Surg 1994;20:751-8.

8 Kakkar VV, Cohen AT, Edmonson RA, et al. Low molecular weight versus standard heparin for prevention of venous thromboembolism after major abdominal surgery. Lancet 1993:341:259-65.

9 Claggett GP, Anderson FA Jr, Heit J, et al. Prevention of venous thromboembolism. Chest 1995; 108:213S-334S

10 Bierkeset $\mathbf{P}$, Larsen $\mathrm{S}$, Reiertsen $\mathrm{O}$. Evaluation of enoxaparin given before and after operation to prevent venous thromboembolism during digestive surgery: play-the-winner designed study. World J Surg 1997;21:584-9.

11 Scurr JH, Coleridge-Smith PD, Hasty JH. Deep venous thrombosis: a continuing problem. BN 1988;297:28-9

12 Kakkar VV Howe CT, Nicolaides AN, et al. Deep vein thrombosis of the leg. Is there a high risk group? Am J Surg 1970;1 20:527-30.

13 Nicolaides AN, Irving D. Clinical factors and the risk of deep venous thrombosis. In: Nicolaides AN, ed. Thromboembolism: aetiology, advances in prevention and management. Lancaster: Medical and Technical Publishing, 1975: 193-204.

14 Lowe GD, Osborne DH, McArdle BM, et al. Prediction and selective prophylaxis of venous thrombosis in elective gastrointestinal surgery. Lancet 1982;i:409-12.

15 Campbell WB, Ridler BMF. Varicose vein surgery and deep vein thrombosis. Br J Surg 1995;82, 1494-7.

16 Guilleband J. Surgery and the pill. BM 1985;291:498-9.

17 Helmrich SP, Rosenberg L, Kaufman DW, et al. Venous thromboembolism in relation to oral contraceptive use. Obstet Gynecol 1987;69:91-5.

18 Notelowitz M, Ware M. Coagulation risks with postmenopausal oestrogen therapy. In: Studd JWW, ed. Progress in obstetrics and gynaecology. Edinburgh: Churchill Livingstone, 1982: 228-40.

19 Carter CJ. Thrombosis in relation to oral contraceptives and hormone replacement therapy. In: Greer IA, Turpie AGG, Forbes CD, eds. Haemostasis and thrombosis in obstetrics and gynaecology. London: Chapman and Hall, 1992: 371-88.

20 Odoom JA, Sih IL. Epidural analgesia and anticoagulant therapy Anaesthesia 1983;38:254-9. 